\title{
Adiponectin inhibits the production of TNF-alpha, IL-6 and chemokines by human lung macrophages
}

\author{
Helene Salvator ${ }^{1}$, Stanislas Grassin-Delyle ${ }^{2}$, Marion Brollo ${ }^{2}$, Louis-jean Couderc ${ }^{2}$, \\ Charlotte Abrial' ${ }^{2}$, Tatiana Victoni², Emmanuel Naline ${ }^{2}$, and Philippe Devillier ${ }^{2}$ \\ ${ }^{1}$ Viorologie Immunologie Moleculaire (VIM) Suresnes - V2I- UMR 0892 \\ ${ }^{2}$ Virologie et Immunologie Moleculaire (VIM) Suresnes, V2I - UMR-0892
}

February 6, 2021

\begin{abstract}
Background and purpose: Obesity is associated with an elevated risk of severe respiratory infections and inflammatory lung diseases. The objectives were to investigate (i) the production of adiponectin by human lung explants, (ii) the expression of the adiponectin receptors AdipoR1 and AdipoR2 by human lung macrophages (LMs), and (iii) the impact of recombinant human adiponectin and a small-molecule APN receptor agonist (AdipoRon) on LMs activation. Experimental approach: Human parenchyma explants and LMs were isolated from patients operated for carcinoma. The LMs were cultured with recombinant adiponectin or AdipoRon and stimulated with LPS (10 ng.mL-1), poly(I:C) (10 $\mu$ g.mL-1) or interleukin(IL)-4 (10 ng.mL-1) for $24 \mathrm{~h}$. Cytokines or adiponectin, released by explants or LMs, were measured using ELISAs. The mRNA levels of AdipoR1 and AdipoR2 were determined using real-time quantitative PCR. AdipoRs expression was also assessed with confocal microscopy. Key results: Adiponectin was released by lung explants at a level negatively correlated with the donor's body mass index. AdipoR1 and AdipoR2 were both expressed in LMs. Adiponectin (3-30 $\mu$ g.mL-1) and AdipoRon (25-50 $\mu$ M) markedly inhibited the LPS- and poly(I:C)-induced release of Tumor Necrosis Factor-alpha, IL-6 and chemokines (CCL3, CCL4, CCL5, CXCL1, CXCL8, CXCL10) and the IL-4-induced release of chemokines (CCL13, CCL17, CCL22) in a concentrationdependent manner. Recombinant adiponectin produced in mammalian cells (lacking low molecular weight isoforms) had no effects on LMs. Conclusions and implications: The low-molecular-weight isoforms of adiponectin and AdipoRon have an antiinflammatory activity in the lung environment. Targeting adiponectin receptors may constitute a new means of controlling airways inflammation.
\end{abstract}

\section{Hosted file}

020121_APN Lung Macrophage BJP.pdf available at https://authorea.com/users/394146/articles/ 507603-adiponectin-inhibits-the-production-of-tnf-alpha-il-6-and-chemokines-by-humanlung-macrophages

\section{Hosted file}

020121_APN Lung MP_Table 1.pdf available at https://authorea.com/users/394146/articles/ 507603-adiponectin-inhibits-the-production-of-tnf-alpha-il-6-and-chemokines-by-humanlung-macrophages 
Figure 1

a)

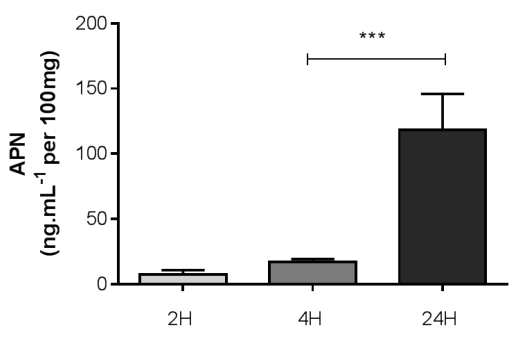

b)

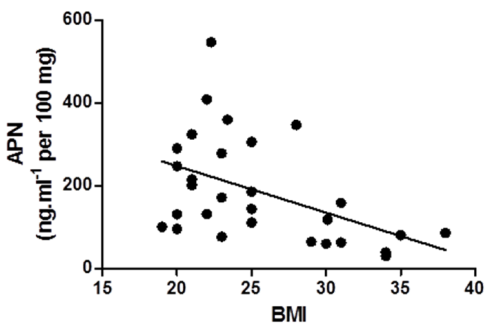


Figure 2

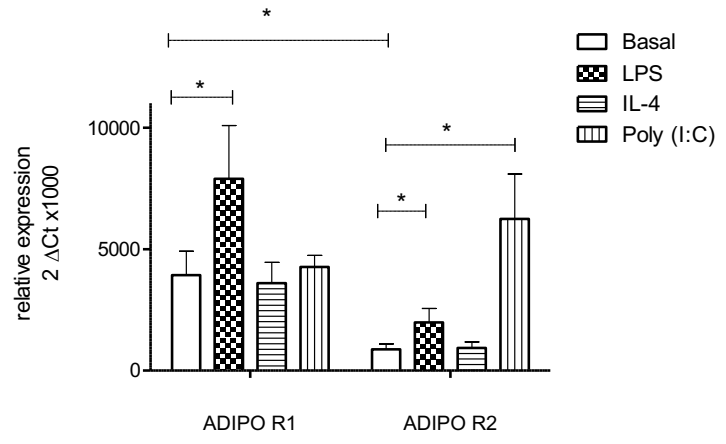




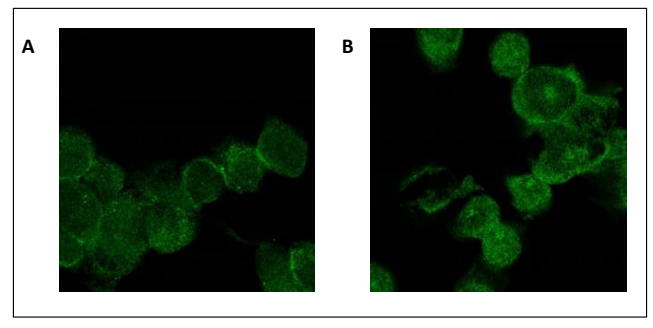



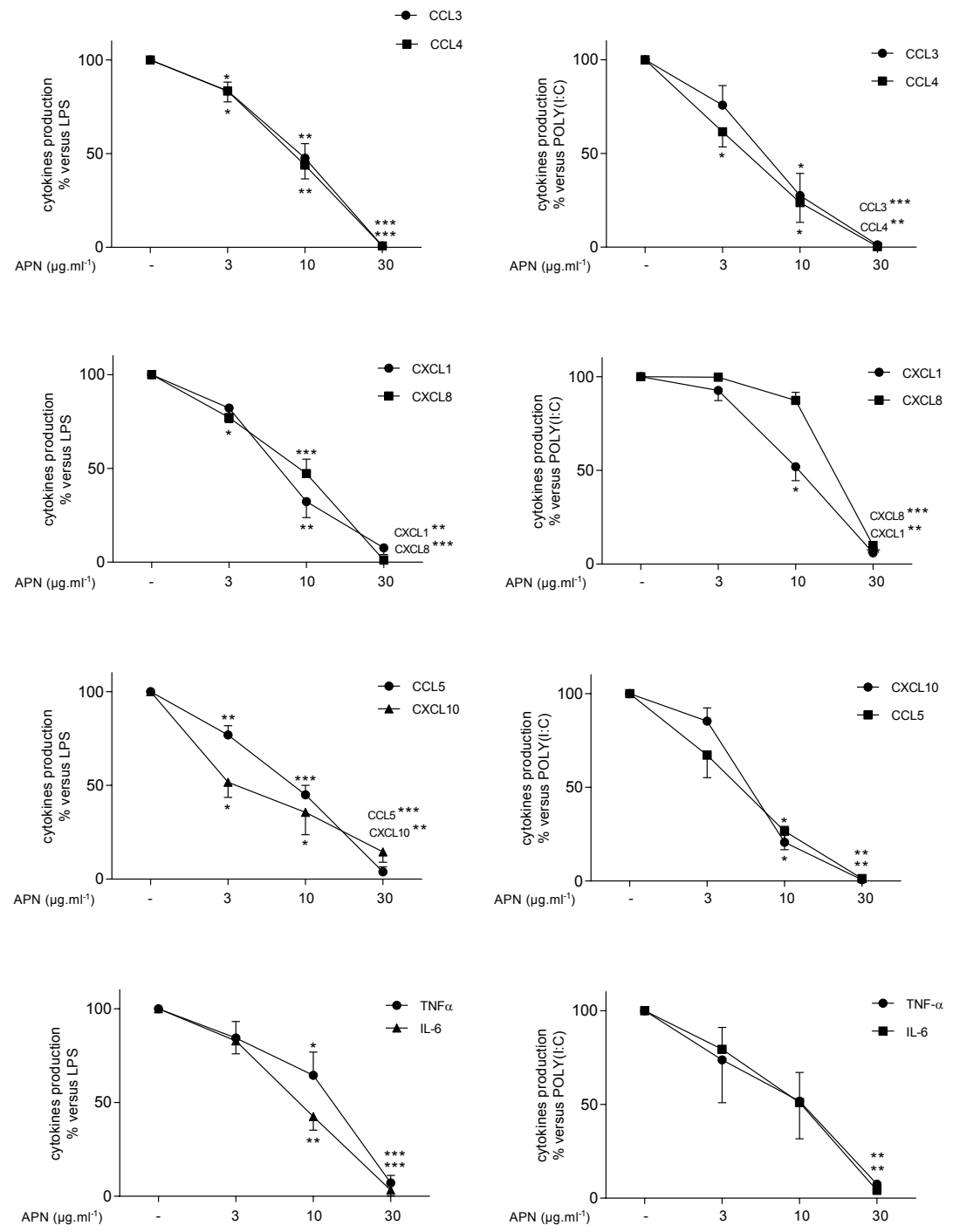

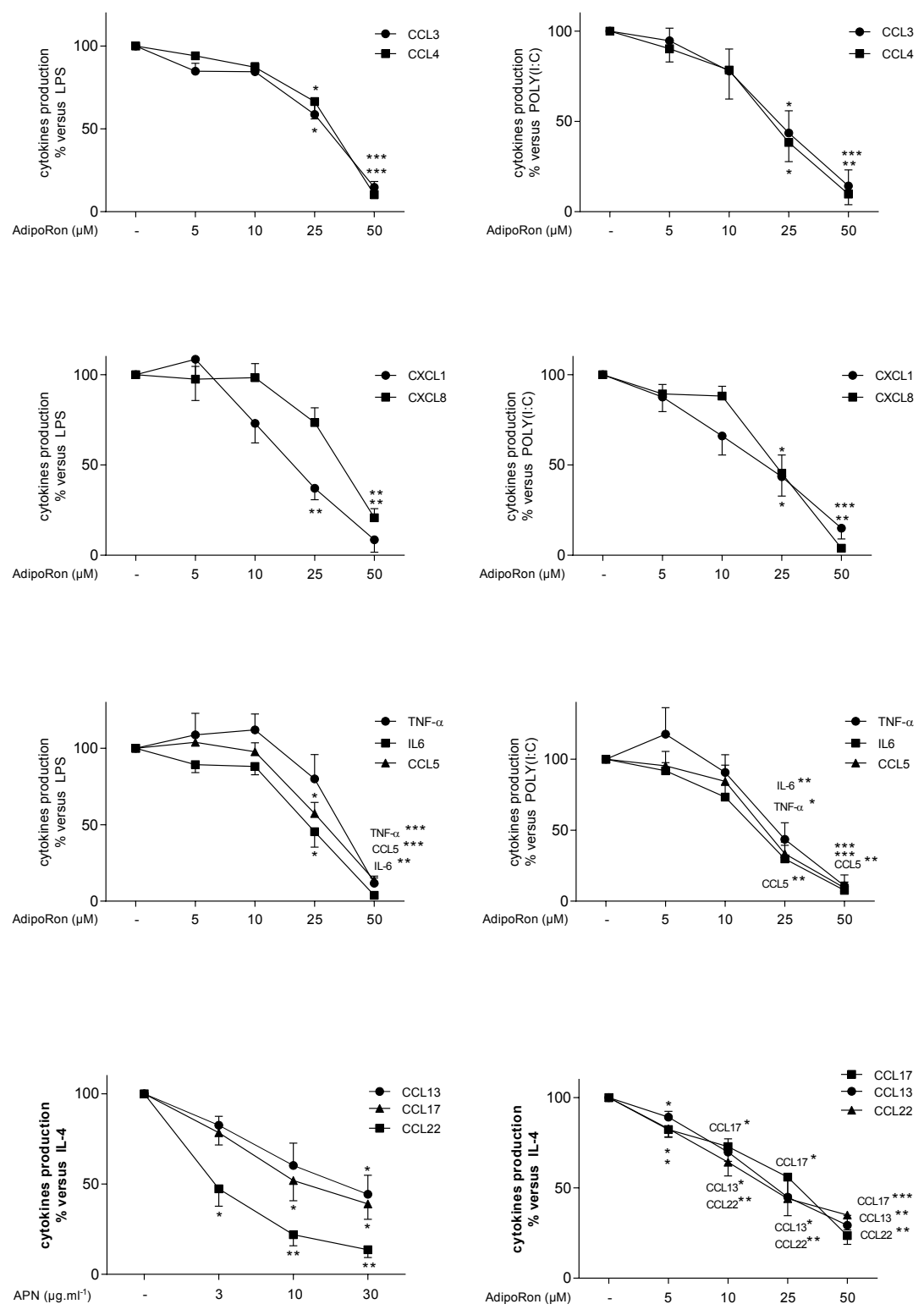